\title{
Fetal and neonatal alloimmune thrombocytopenia
}

INSERM

\section{Source}

INSERM. (1999). Orphanet: an online rare disease and orphan drug data base. Fetal and neonatal alloimmune thrombocytopenia. ORPHA:853

Foetal/neonatal alloimmune thrombocytopaenia (NAIT) results from maternal alloimmunisation against foetal platelet antigens inherited from the father and different from those present in the mother, and usually presents as a severe isolated thrombocytopaenia in otherwise healthy newborns. 